\title{
Hierarquização multicritério de alternativas de macrodrenagem urbana na bacia hidrográfica da Estrada Nova em Belém-PA, Amazônia, Brasil
}

\author{
Multicriteria hierarchy of urban macrodrainage alternatives in the Estrada Nova watershed in \\ Belém-PA, Amazonia, Brazil \\ Jerarquía multicriterio de alternativas de macrodrenaje urbano en la cuenca de Estrada Nova en \\ Belém-PA, Amazonia, Brasil
}

Recebido: 12/08/2021 | Revisado: 17/08/2021 | Aceito: 21/08/2021 | Publicado: 23/08/2021

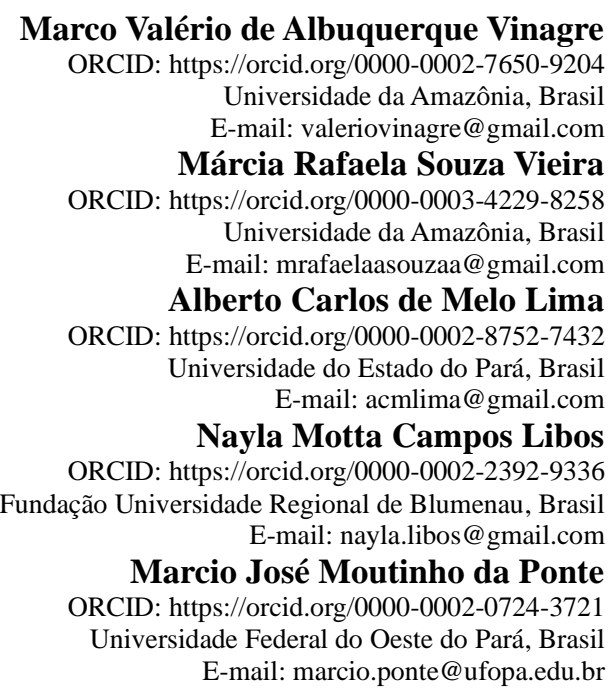

\begin{abstract}
Resumo
O objetivo deste trabalho foi hierarquizar alternativas de macrodrenagem urbana na Sub-bacia 2 da Bacia Hidrográfica da Estrada Nova (BHEN) em Belém-PA, Amazônia, Brasil, para auxílio na seleção de alternativa a ser implantada. A metodologia utilizada considerou critérios técnicos, ambientais e econômicos, e foi aplicada a cinco alternativas possíveis para a funcionalidade da Sub-bacia em estudo, as quais foram desenvolvidas com o uso do código computacional livre Storm Water Management Model (SWMM). Na modelagem considerou-se precipitação de 50 anos de retorno, marés, comprimentos, seções dos canais, galerias e pontes existentes, coeficientes de Manning de revestimentos, disponibilidade de áreas para implantação de componentes necessários à funcionalidade do sistema, como novos canais, unidades de armazenamento e comportas. Dentre as cinco alternativas a segunda foi a mais bem classificada, essa considera concordância de seções e unidade de armazenamento. Desta forma, os resultados obtidos foram muito promissores e confirmaram a expectativa pelo uso de melhores práticas de manejo de águas pluviais, tendo a metodologia utilizada permitido a resolução do problema muito satisfatoriamente.
\end{abstract}

Palavras-chave: Drenagem urbana; Manejo de águas pluviais; Análise multicritério; SWMM.

\begin{abstract}
The objective of this work was to rank urban macrodrainage alternatives in Sub-basin 2 of the Estrada Nova Hydrographic Basin (BHEN) in Belém-PA, Amazonia, Brazil, to aid in the selection of an alternative to be implemented. The methodology used considered technical, environmental and economic criteria, and was applied to five possible alternatives for the functionality of the Sub-basin under study, which were developed using the free computational code Storm Water Management Model (SWMM). The modeling considered precipitation of 50 years of return, tides, lengths, sections of channels, existing galleries and bridges, Manning coefficients, availability of areas to implement components necessary for the system's functionality, such as new channels, storage units and floodgates. Amongst the five alternatives, the second alternative resulted in the best ranking, which considers agreement of sections and storage unit. Thus, the results obtained were very promising and confirmed the expectation for the use of better rainwater management practices, with the methodology used allowing the problem to be solved very satisfactorily.
\end{abstract}

Keywords: Urban drainage; Stormwater management; Multicriteria analysis; SWMM. 


\begin{abstract}
Resumen
El objetivo de este trabajo fue clasificar las alternativas de macrodrenaje urbano en la Subcuenca 2 de la Cuenca Hidrográfica de Estrada Nova (BHEN) en Belém-PA, Amazonia, Brasil, para ayudar en la selección de una alternativa a implementar. La metodología utilizada consideró criterios técnicos, ambientales y económicos, y se aplicó a cinco posibles alternativas para la funcionalidad de la Subcuenca en estudio, las cuales fueron desarrolladas utilizando el código computacional libre Storm Water Management Model (SWMM). La modelación consideró precipitación de 50 años de retorno, mareas, longitudes, tramos de canales, galerías y puentes existentes, coeficientes de Manning de revestimientos, disponibilidad de áreas para la implementación de componentes necesarios para la funcionalidad del sistema, tales como nuevos canales, unidades. de almacenaje y compuertas. Entre las cinco alternativas, la segunda fue la mejor clasificada, que considera la concordancia de secciones y unidad de almacenamiento. Así, los resultados obtenidos fueron muy prometedores y confirmaron la expectativa por el uso de mejores prácticas de manejo del agua de lluvia, con la metodología empleada permitiendo resolver el problema de manera muy satisfactoria.
\end{abstract}

Palabras clave: Drenaje urbano; Gestión de aguas pluviales; Análisis multicriterio; SWMM.

\title{
1. Introdução
}

Grandes alagamentos infelizmente ocorrem com frequência em grandes cidades, evidenciando a necessidade de planejamento e gestão competentes das águas urbanas, inclusive com a busca de novas alternativas de prevenção e minimização desses eventos. A falta de planejamento no crescimento dos centros urbanos acarreta diversos eventos que afetam a vida da população como deslizamentos de terra, alagamentos e formação de ilhas de calor, isso porque não ponderam parâmetros de qualidade ambiental. (Abreu \& Andrade, 2019, p. 30). Correa (2017) ressalta que a falta de planejamento urbano na cidade juntamente com o rápido crescimento populacional, são fatores que influenciam nos impactos sociais devido a ocorrência de eventos como alagamentos.

Historicamente no Brasil, a urbanização aconteceu sem nenhum controle ou presença de planejamento urbano, e o resultado disso foram ocupações sem o mínimo de ordenamento, cenários como impermeabilização do solo, modificações da topografia natural, retificação dos cursos d'água, a não consideração do escoamento pluvial, todas essas condições trazem problemas ambientais para as cidades (Silva, 2016).

Os problemas associados ao escoamento das águas pluviais estão entre os mais preocupantes do desenvolvimento urbano, visto que o processo de urbanização altera significativamente os processos de escoamento natural da bacia hidrográfica, isso ocorre devido ao aumento do escoamento superficial e das vazões de pico dos corpos d'água, diminuição da recarga subterrânea, degradação da qualidade da água e da geração de sedimentos (Villanueva, Tassi, Piccilli, Bemfica \& Tucci, 2011).

No mesmo sentido Canholi (1999) afirma que um dos maiores desafios da gestão pública é solucionar problemas relacionados ao crescimento urbano, uma vez que a ocupação desordenada resulta na impermeabilização crescente e excessiva do solo, além da ocorrência de práticas comuns e muitas vezes desnecessárias como a canalização de rios e córregos.

O aumento do escoamento superficial acaba por sobrecarregar os sistemas de drenagem que, de acordo com Ferreira (2019), normalmente são projetados para um determinado tempo de retorno de chuva, mas a capacidade do sistema de drenagem não suporta esse grande pico de vazão causado pela impermeabilização do solo na expansão urbana, e isso favorece processos de alagamentos. Portanto, para a minimização do pico do hidrograma unitário, muitos municípios acabam por instalarem mecanismos de retenção de água, como os reservatórios de retenção e as bacias subterrâneas. Ainda, recorrem à ampliação do sistema de macrodrenagem.

A cidade de Belém é marcada por alagamentos recorrentes isso porque possui bacias hidrográficas extremamente urbanizadas e de forma inadequada, algumas bacias apresentam zonas críticas de ocorrência desses eventos, como a Sub-bacia 2 da Bacia Hidrográfica da Estrada (BHEN), a qual, segundo o Plano Diretor Municipal (Belém, 2008), localiza-se na Zona de Uso Misto ZUM-8 de Alta Densidade II (ZAD II), apresentando-se com as áreas mais povoadas e densas da cidade, com 
ocupação acima de 95\% dos lotes, essas características contribuem para a sobrecarga das bacias hidrográficas.

O presente estudo procura responder a seguinte pergunta-chave: É possível utilizar análise multicriterial baseada em aspectos técnicos, econômicos e ambientais para hierarquizar e selecionar a melhor dentre as cinco alternativas estudadas, as quais foram desenvolvidas com modelagem computacional?

\section{Metodologia}

\subsection{Análise Multicritério}

A Análise Multicritério consiste em um conjunto de técnicas que tem como propósito, auxiliar pessoas e/ou organizações na tomada de decisões acerca de um problema, em situações nas quais é necessário identificar prioridades, considerando, ao mesmo tempo, diversos aspectos (Jannuzzi, Miranda \& Silva, 2006).

A seleção de alternativas de projetos as avaliações não devem ser efetuadas a partir da consideração de apenas um critério segundo Castro (2002), para o autor em alguns casos, é importante considerar diversos aspectos ao mesmo tempo como econômicos, sociais, ambientais, políticos assim como quaisquer outros que se mostrarem relevantes à comparação de um determinado conjunto de alternativas.

Para Castro (2007) a análise de alternativas de projeto deve ir além da comparação de apenas critérios técnicos ou econômicos. Questões políticas, sociais e ambientais, além de outras que forem relevantes ao processo, podem ser consideradas, de forma a torná-la mais abrangente e consistente.

A metodologia do estudo baseou-se em análise de multicritérios segundo Mendonça (2009) e Brito (2006) para a seleção da melhor alternativa considerando aspectos técnicos, econômicos e ambientais, a fim de minimizar os problemas de alagamentos na sub-bacia 2 da BHEN.

Para a hierarquização das alternativas na análise assumiu-se valores que estão relacionados a mensuração dos aspectos, portanto, as pontuações adotadas foram as seguintes:

$$
\begin{aligned}
& \text { Alta }=10 ; \\
& \text { Médio }=5 ; \\
& \text { Baixo= } 0 .
\end{aligned}
$$

O resultado da classificação final é obtido pela equação:

$$
\sum \frac{P_{\alpha} \cdot V_{\alpha}}{P}
$$

Onde:

$P=$ Peso;

$P_{\mathscr{Q}}=$ Peso do aspecto;

$V_{\varangle}=$ Valor do aspecto na alternativa.

Em seguida, seleciona-se a alternativa que possui o menor valor, para Brito (2006) essa alternativa possui maior proximidade da solução ideal.

\subsection{Local de Estudo}

Este estudo foi realizado na cidade de Belém, que possui uma área territorial correspondente a $1.059,458 \mathrm{~km}^{2} \mathrm{e}$ população estimada em 2019 de 1.492 .745 pessoas, com densidade demográfica de 1.315,26 hab./km², de acordo com dados o 
Instituto Brasileiro de Geografia e Estatística (IBGE) (2011 e 2019). A Figura 1 a seguir apresenta a localização da cidade de Belém-PA e a hipsometria da BHEN.

Figura 1. Localização da cidade de Belém do Pará, na Amazônia Brasileira.

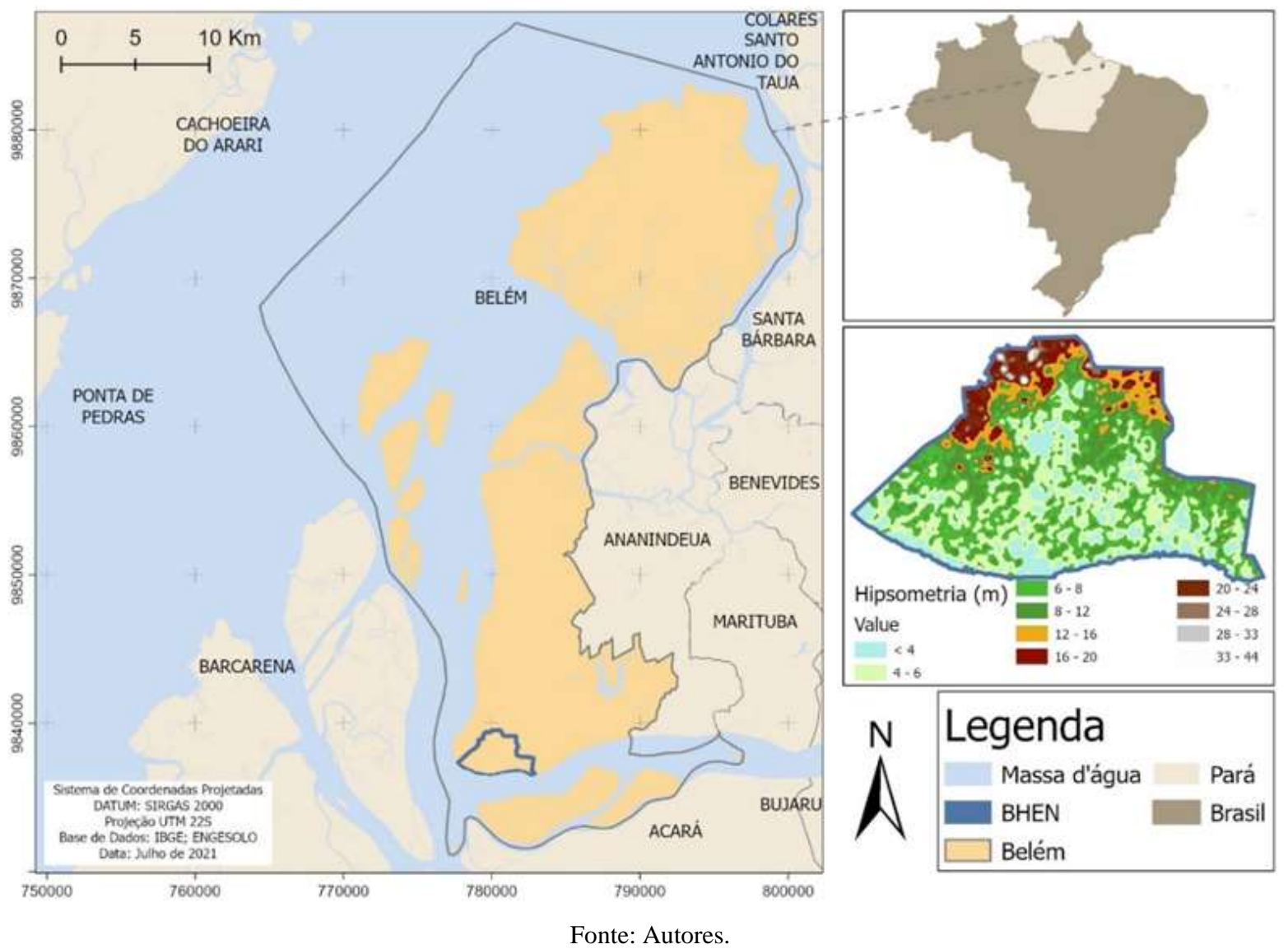

A cidade, cercada por rios, possui uma topografia com cotas baixas e pouco ondulada. Parte significativa de Belém e da BHEN encontra-se a uma altitude de até 4 metros.

Observa-se na Figura 2 a Sub-bacia 2 da BHEN, tema de estudo deste artigo, delimitada pelas vias: Av. Nazaré, Rua Eng. Fernando Guilhon, Trav. 14 de Março, Av. Alcindo Cacela, Av. Roberto Camelier e Av. Bernardo Sayão com o Rio Guamá. 
Figura 2. Delimitação da Sub-bacia 2 na BHEN.

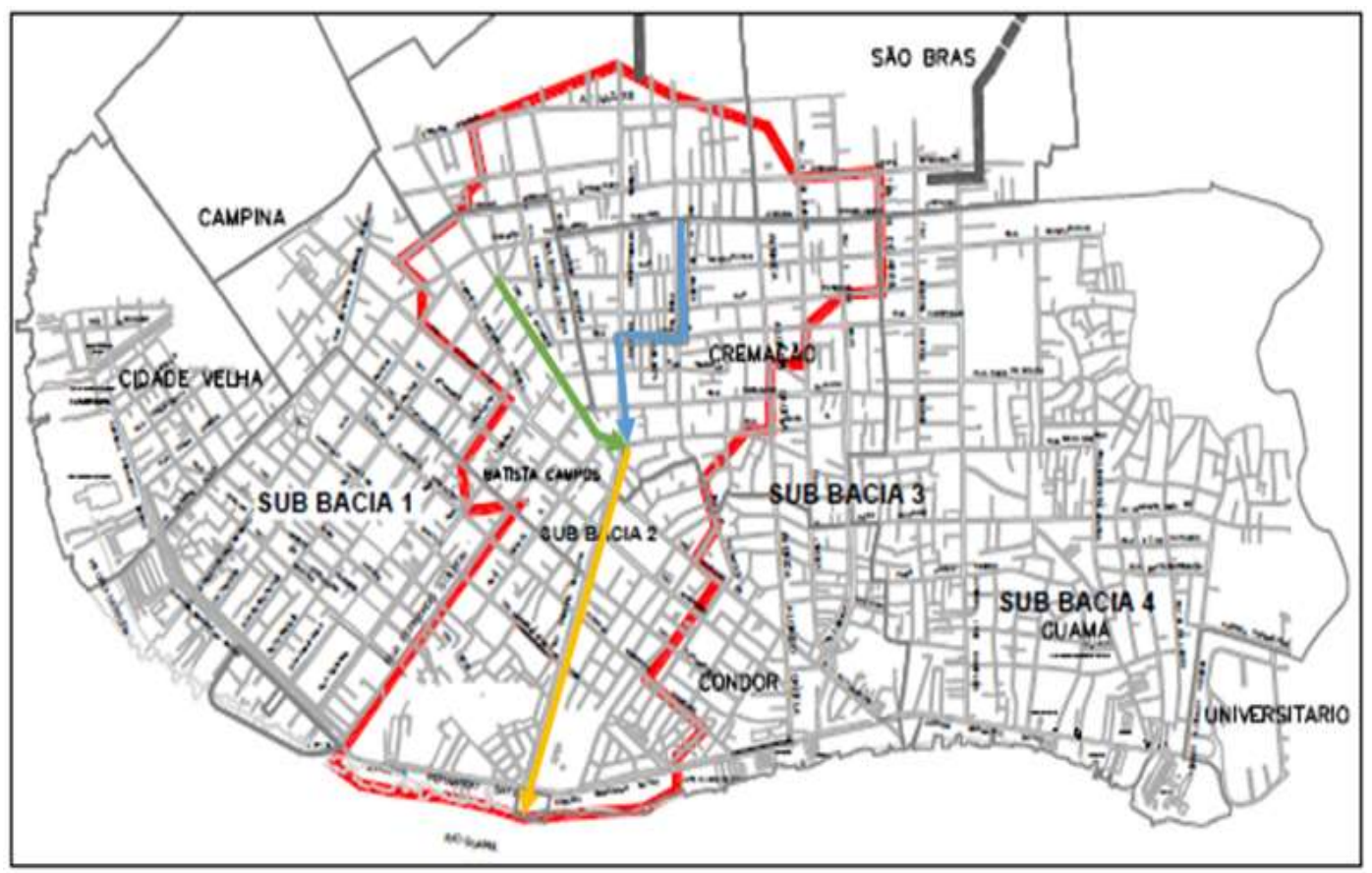

Fonte: ENGESOLO (2008).

A Sub-bacia 2 da BHEN consiste em uma área totalmente urbanizada, em sua maioria por residências unifamiliares, com elevada percentagem de quintais e jardins.

Os Canais estudados e a localização dentro da Sub-bacia 2 são:

Canal 1 - Tv. Doutor Morais e Rua São Miguel (cor verde na figura)

Canal 2 - Tv. 14 de Março, Rua dos Caripunas, Av. Generalíssimo e Passagem Pinheiro (cor azul na figura).

Canal 3 - Tv. Quintino Bocaiúva (cor amarela na figura)

Os Canais 1 e 2 apresentam alagamentos recorrentes devido a existência de trecho intermediário não escavado e nem revestido, com fundo elevado, que se constitui em obstáculo ao escoamento, na junção dos canais da Rua Dr. Moraes/Rua São Miguel e Av. Generalíssimo/Passagem Pinheiro com o canal da Tv. Quintino Bocaiuva.

O Sistema de Macrodrenagem da Sub bacia 2 da BHEN apresenta trechos de canais implantados e a implantar, sendo necessário interligar os trechos já revestidos de montante da Rua Dr. Moraes e da Av. Generalíssimo Deodoro com os de jusante da Tv. Quintino Bocaiúva. A Figura 3 a seguir mostra imagem de satélite com o trecho de interligação não executado. 
Research, Society and Development, v. 10, n. 11, e76101119352, 2021

(CC BY 4.0) | ISSN 2525-3409 | DOI: http://dx.doi.org/10.33448/rsd-v10i11.19352

Figura 3. Trecho de interligação não executado.
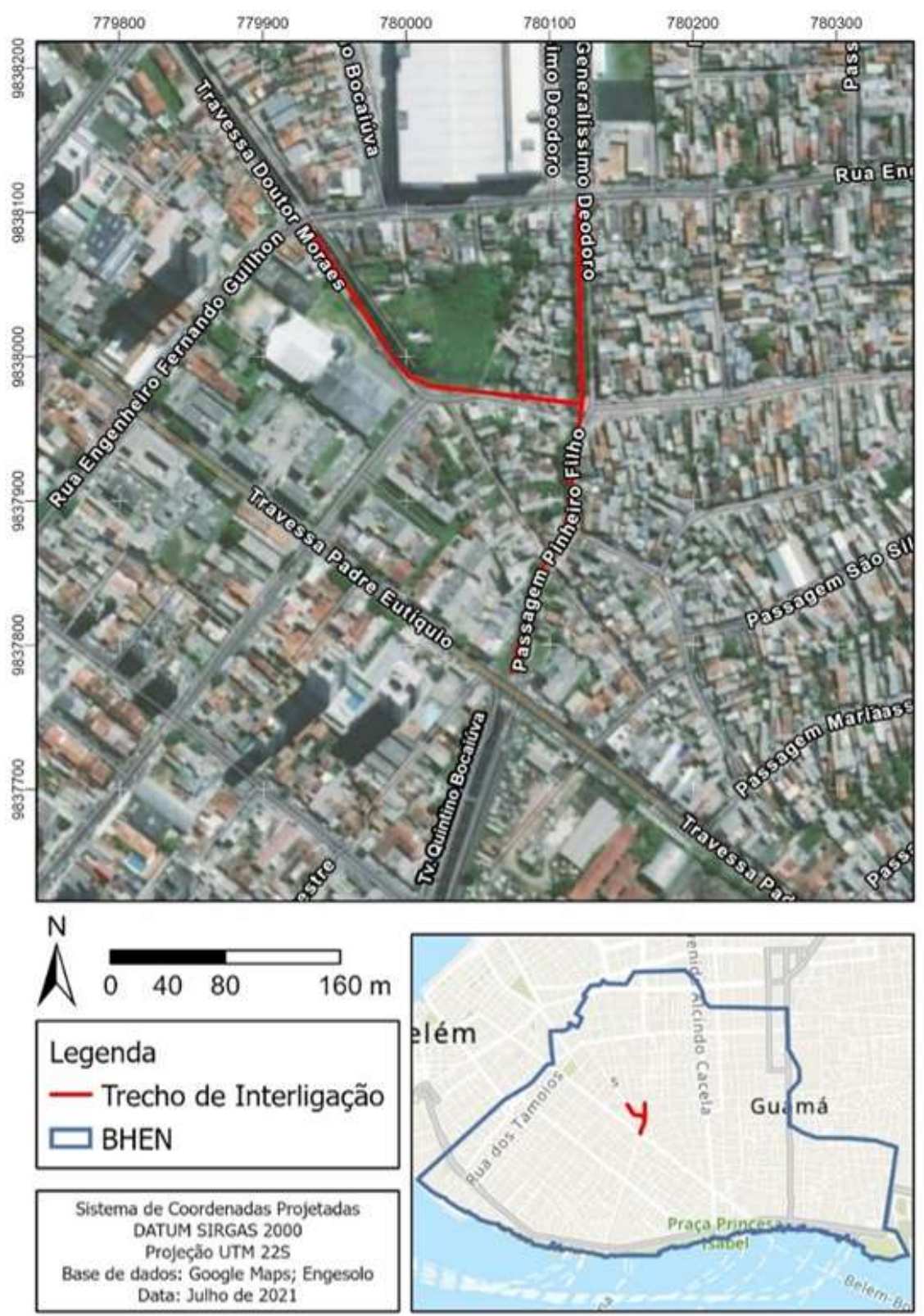

Fonte: Autores.

Observa-se na figura acima que a interligação dos trechos de canais de montante, ou seja, Rua Dr. Moraes e Av. Generalíssimo Deodoro, com o de jusante, Tv. Quintino Bocaiuva, apresenta-se em terreno natural, sem escavação e sem revestimento, constituindo-se em importante obstáculo ao escoamento da Sub-bacia 2 da BHEN. A Figura 4 exibe a Rua Engenheiro Fernando Guilhon entre Trav. Quintino Bocaiúva e Av. Generalíssimo Deodoro completamente alagada, local próximo ao trecho de interligação. 
Research, Society and Development, v. 10, n. 11, e76101119352, 2021

(CC BY 4.0) | ISSN 2525-3409 | DOI: http://dx.doi.org/10.33448/rsd-v10i11.19352

Figura 4. Alagamento da Rua Engenheiro Fernando Guilhon.

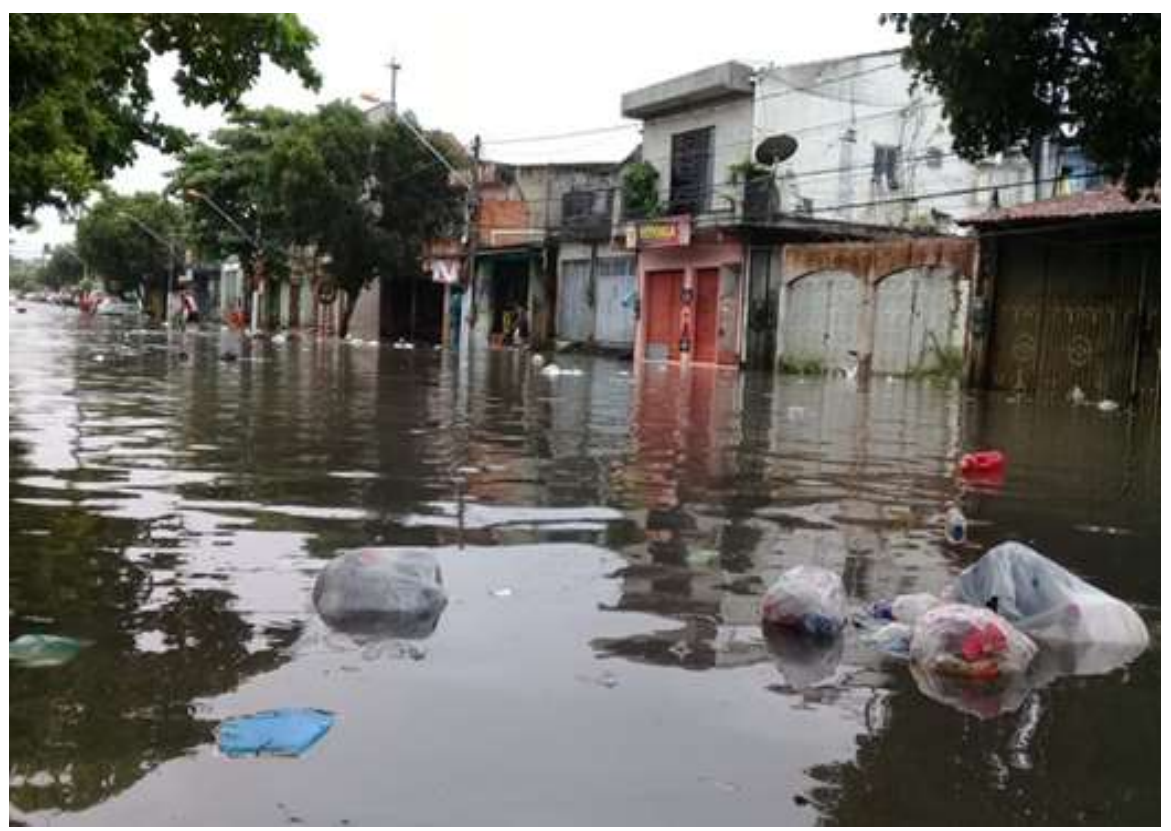

Fonte: Souza (2020).

O local apresentado na Figura 4 fica aproximadamente a $150 \mathrm{~m}$ do trecho de interligação não executado. A Figura 5 a seguir apresenta esquematicamente modelagem da Sub-bacia 2 da BHEN no SWMM.

Figura 5. Esquema da modelagem da Sub-bacia 2 da BHEN no SWMM.

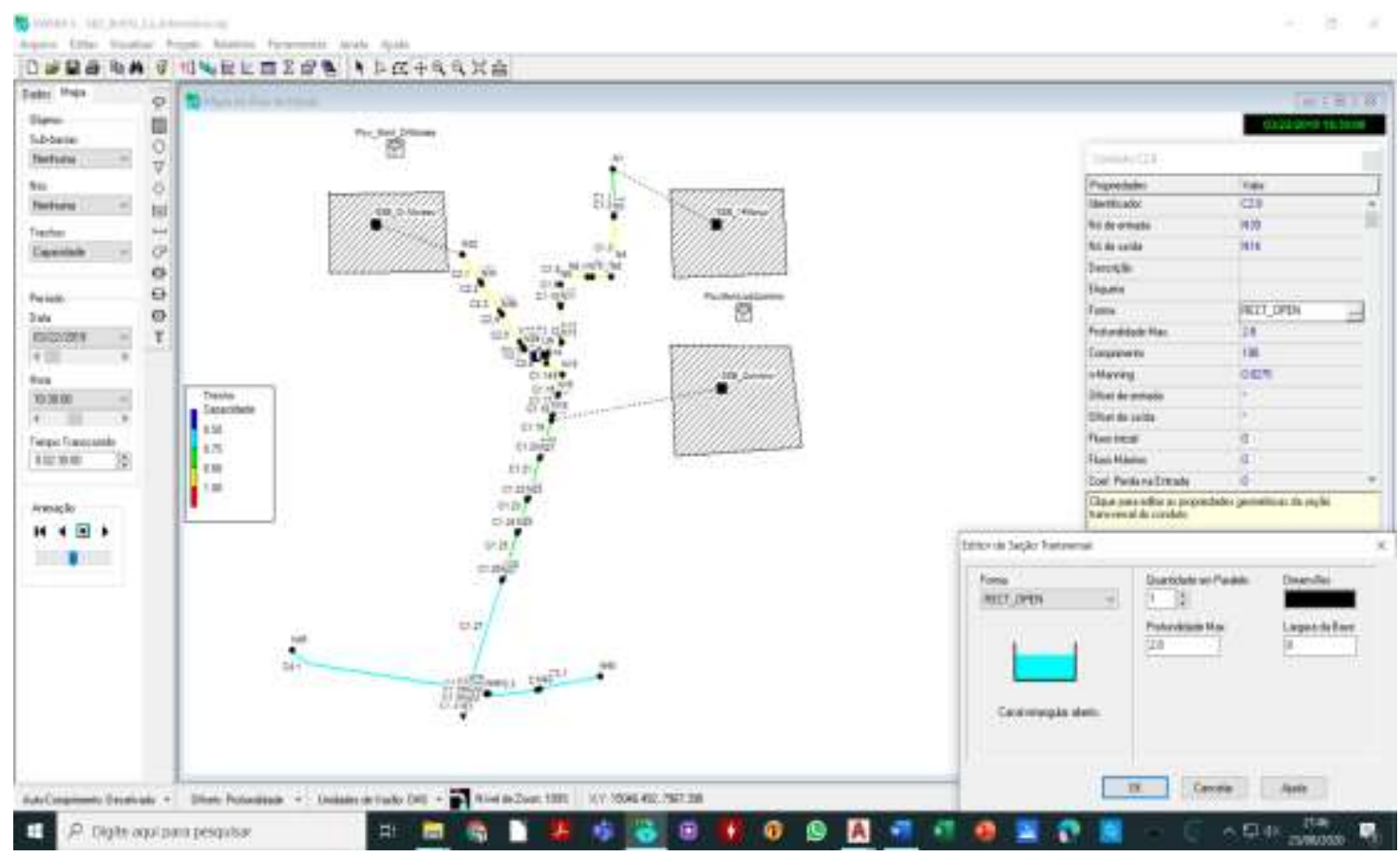

Fonte: Autores, com uso do SWMM (2021).

Observa-se na Figura acima as três subdivisões que constituem a Sub-bacia 2, cujas áreas estão explicitadas a seguir, na Tabela 1 . 
Research, Society and Development, v. 10, n. 11, e76101119352, 2021

(CC BY 4.0) | ISSN 2525-3409 | DOI: http://dx.doi.org/10.33448/rsd-v10i11.19352

Tabela 1. Principais características das subdivisões da Sub-bacia 2 da BHEN.

\begin{tabular}{|c|c|c|c|}
\hline \multirow{2}{*}{$\begin{array}{l}\text { Característica } \\
\text { hidrográfica }\end{array}$} & \multicolumn{3}{|c|}{ Subdivisões } \\
\hline & $\begin{array}{l}\text { Montante Rua Dr. } \\
\text { Moraes }\end{array}$ & $\begin{array}{c}\text { Montante Tv. Quintino } \\
\text { Bocaiúva }\end{array}$ & Jusante Tv. Quintino Bocaiúva \\
\hline Área de Drenagem $\left(\mathrm{km}^{2}\right)$ & 0,991 & 1,386 & 1,663 \\
\hline$\%$ da Área & 25 & 34 & 41 \\
\hline $\begin{array}{l}\text { Percurso do Escoamento } \\
\text { (m) }\end{array}$ & 1.565 & 2.060 & 1.820 \\
\hline $\begin{array}{l}\text { Tempo de concentração } \\
\text { (min) }\end{array}$ & 100 & 120 & 150 \\
\hline Curve Number (SCS) & 0,85 & 0,85 & 0,85 \\
\hline
\end{tabular}

Uso e Ocupação

85 a $95 \%$ das áreas urbanizadas - poucas áreas verdes

Fonte: Adaptado de "Elaboração de estudos e projetos básicos necessários à preparação do programa de macrodrenagem da Bacia da Estrada Nova" de ENGESOLO Engenharia LTDA. Belo Horizonte, 2008; e "Projeto do Sistema de Macrodrenagem: Reprogramação das Metas do Contrato de Repasse n⿳0 228.498-26/2008 FGTS/CAIXA/PAC Sub-bacia 02 da Bacia Hidrográfica da Estrada Nova" de APCE - ASSESSORIA, PROJETOS E CONSULTORIA LTDA, 2016.

Observa-se na Tabela 1 os dados das três divisões da Sub-bacia 2 em três unidades menores, utilizados nos estudos hidrológicos e hidráulicos desenvolvidos neste trabalho.

As precipitações críticas apresentam sua distribuição espacial uniforme em cada uma das três divisões da Sub-bacia 02 da BHEN. A precipitação utilizada na simulação tem o tempo de recorrência de cinquenta anos e duração de quatro horas, conforme mostra-se na Figura 6 a seguir, a qual apresenta as séries temporais de período de retorno de cinquenta anos, para uso no SWMM, acopladas aos pluviômetros referentes às três subdivisões da Sub-bacia 02, e a curva de maré utilizada na simulação.

Figura 6. Séries temporais das divisões da Sub-bacia 02 da BHEN, ambas com período de retorno de 50 anos e curva da maré.
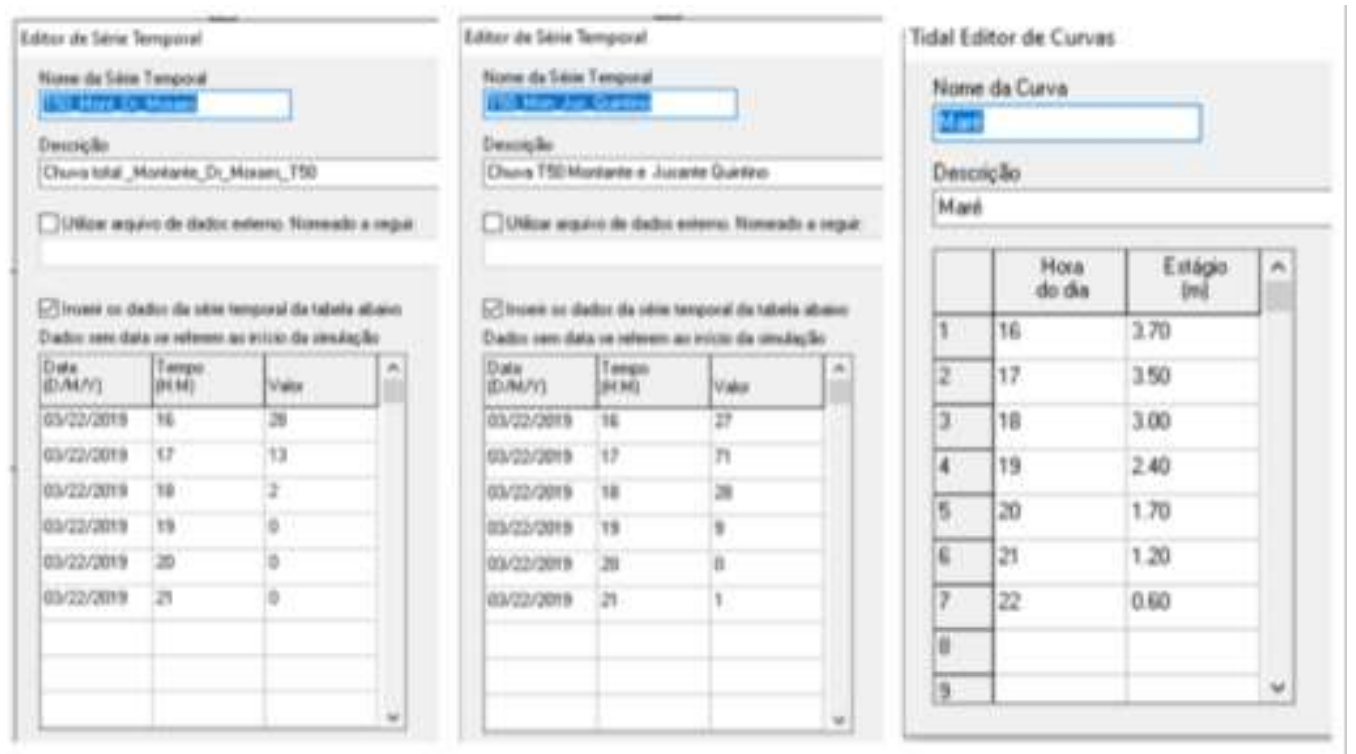

Fonte: Autores (2021).

Observam-se na Figura 6 acima as séries temporais de precipitações de período de retorno de 50 anos, utilizadas nos 
estudos com o SWMM. São duas séries temporais, uma para sub-bacia montante Dr. Moraes, e outra para as sub-bacias montante e jusante Tv. Quintino Bocaiúva devido ao fato da chuva ser a mesma para ambas. Para estudar o evento iniciando com a preamar e o comportamento do sistema até a baixamar, portanto evento com duração de seis horas, incluiu-se a precipitação das próximas duas horas. Observa-se que a curva de maré da Figura 6 acima apresenta seu início na preamar e final na baixa-mar, com duração de seis horas.

\subsection{Alternativas Estudadas}

As cinco alternativas estudadas foram desenvolvidas com o uso do código computacional livre SWMM, para o estudo do comportamento hidráulico dos canais da Sub-bacia 2 da BHEN. O SWMM é um modelo dinâmico chuva-vazão que simula a quantidade e qualidade do escoamento superficial, especialmente em áreas urbanas. A simulação no software requereu dados pluviométricos obtidos no Sistema Nacional de Recursos Hídricos (SNRH) e foi considerado para o estudo uma série temporal com período de retorno de cinquenta anos.

A Sub-bacia 2 da BHEN possui características importantes que foram consideradas na simulação como a área e o grau de ocupação do local interferindo diretamente no Curve Number, o parâmetro que retrata o grau de impermeabilização do solo. Também foram necessários os comprimentos e geometria das seções dos canais, galerias e pontes existentes, e os coeficientes de Manning segundo as características de seus revestimentos. Após a seleção dos parâmetros necessários, foram elaboradas com o uso do SWMM cinco soluções alternativas para a macrodrenagem da Sub-bacia 2 da BHEN.

Na Figura 7 a seguir observa-se que quatro alternativas utilizam a interligação dos canais de montante com os de jusante com concordância de seções, ou seja, sugeriu-se a implantação na Rua Dr. Moraes da Rua Fernando Guilhon até Avenida Generalíssimo, de um conduto com extensão de 198 m, seção retangular aberta, com largura de 8 m e revestimento lateral em concreto, profundidade $2,80 \mathrm{~m}$, fundo em terreno natural. Para a quinta alternativa foi sugerido a concordância de seções no trecho de Implantação na Tv. Quintino Bocaiuva, da Tv. Padre Eutíquio até Av. Bernardo Sayão, de alargamento do canal existente na extensão de $1.275 \mathrm{~m}$, alargamento este coberto com laje, largura de $3 \mathrm{~m}$, altura útil de 2,80 m, em paralelo ao canal existente. 
Research, Society and Development, v. 10, n. 11, e76101119352, 2021

(CC BY 4.0) | ISSN 2525-3409 | DOI: http://dx.doi.org/10.33448/rsd-v10i11.19352

Figura 7. Esquema das cinco alternativas de macrodrenagem para a Sub-bacia 2.

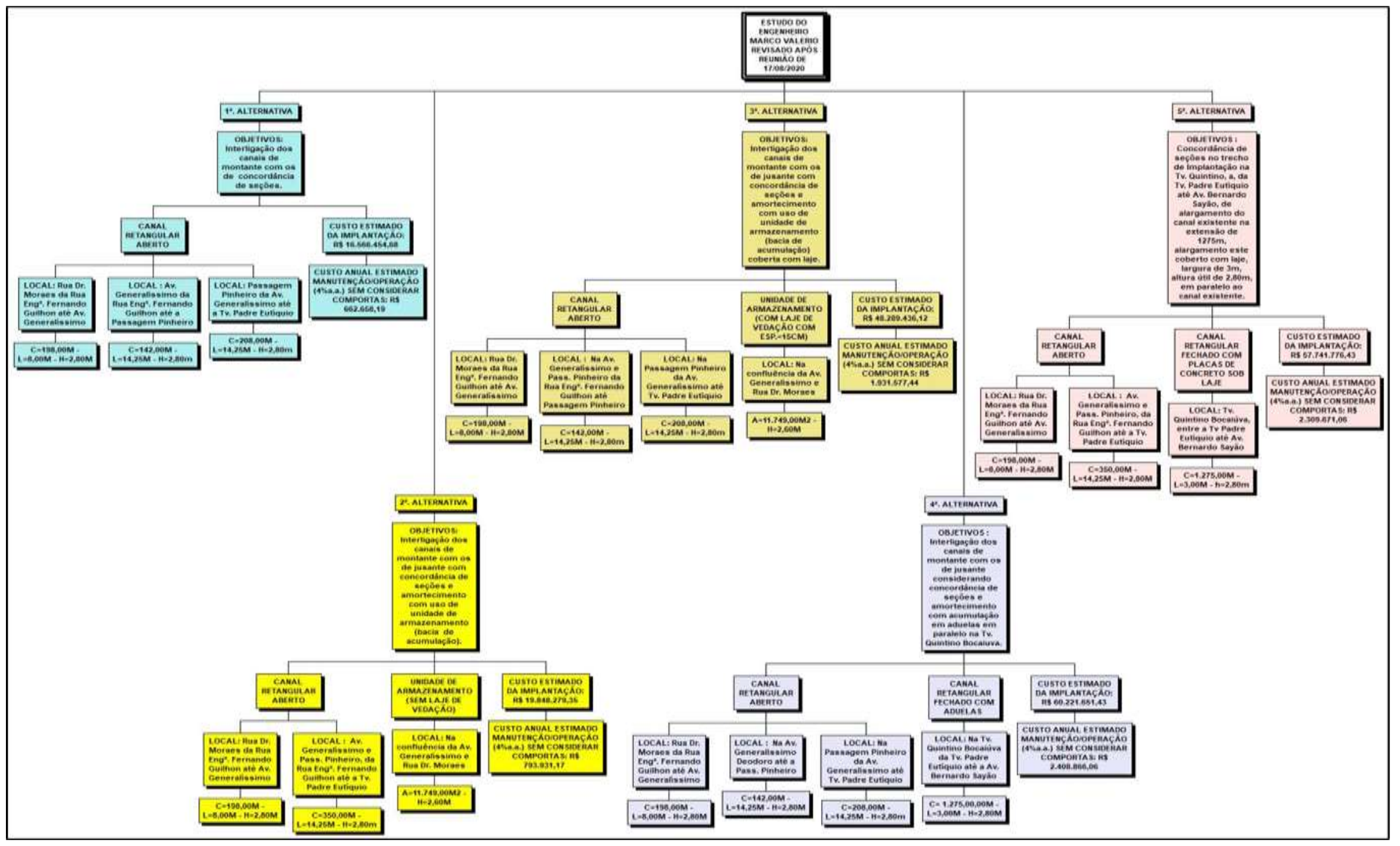

Fonte: Autores. 
O Tabela 2 a seguir sintetiza características das alternativas em estudo, conforme os resultados das simulações efetuadas com o uso do SWMM. Segundo Teixeira, Rodrigues, Crispim, Fernandes e Bittencourt (2020) os processos de avaliação devem conter comparações entre as alternativas a fim de obter as vantagens e desvantagens de cada alternativa estudada em relação aos objetivos já definidos.

Tabela 2. Características das alternativas em estudo.

\begin{tabular}{|c|c|c|c|c|c|}
\hline Alternativas & Ação & Amortecimento & Transbordamentos & Vantagens & Desvantagens \\
\hline 1 & $\begin{array}{l}\text { Interligação com } \\
\text { concordância de } \\
\text { canais }\end{array}$ & Sem & Sim & Menor área & $\begin{array}{l}\text { Dificuldade de manutenção, } \\
\text { risco de lançamento de } \\
\text { esgotos, risco por falha de } \\
\text { comportas }\end{array}$ \\
\hline 2 & $\begin{array}{l}\text { Interligação com } \\
\text { concordância de } \\
\text { canais } \\
\end{array}$ & $\begin{array}{c}\text { Unidade de } \\
\text { armazenamento } \\
\text { aberta } \\
\end{array}$ & Não & $\begin{array}{c}\text { Menor } \\
\text { dificuldade de } \\
\text { manutenção } \\
\end{array}$ & $\begin{array}{c}\text { Maior área, risco por falha } \\
\text { de comportas e lançamento } \\
\text { de esgotos }\end{array}$ \\
\hline 3 & $\begin{array}{l}\text { Interligação com } \\
\text { concordância de } \\
\text { canais }\end{array}$ & $\begin{array}{c}\text { Unidade de } \\
\text { armazenamento } \\
\text { coberta com laje }\end{array}$ & Não & $\begin{array}{c}\text { Menor risco } \\
\text { de lançamento } \\
\text { de esgotos } \\
\end{array}$ & \multirow{3}{*}{$\begin{array}{l}\text { Maior área, risco por falha } \\
\text { de comporta, dificuldade de } \\
\text { manutenção. }\end{array}$} \\
\hline 4 & $\begin{array}{l}\text { Interligação com } \\
\text { concordância de } \\
\text { canais }\end{array}$ & $\begin{array}{l}\text { Acumulação em } \\
\text { aduelas }\end{array}$ & Não & $\begin{array}{c}\text { Médio risco } \\
\text { de lançamento } \\
\text { de esgotos } \\
\end{array}$ & \\
\hline 5 & $\begin{array}{c}\text { Concordância de } \\
\text { canais }\end{array}$ & $\begin{array}{l}\text { Alargamento de } \\
\text { canal sob laje }\end{array}$ & Não & $\begin{array}{c}\text { Médio risco } \\
\text { de lançamento } \\
\text { de esgotos }\end{array}$ & \\
\hline
\end{tabular}

Fonte: Autores.

Por fim, realiza-se a hierarquização geral das alternativas segundo critérios técnicos, econômicos e ambientais, tendo sido atribuídos pesos de acordo com sua importância na efetividade do projeto. Desta forma, para o critério técnico atribui-se peso 3, para o critério econômico também foi atribuído peso 3. Para o critério ambiental, atribuiu-se o peso 4, pela relevância desse tema. O resultado da hierarquização geral apresenta-se a seguir.

\section{Resultados e Discussão}

Os diferentes aspectos considerados em cada viés podem levar a resultados conflitantes, de modo que a solução mais econômica pode não ser a melhor ambiental, ou vice-versa. Assim, os pontos positivos e negativos, as vantagens e desvantagens das alternativas estudadas foram considerados, sistematizados, tratados e ponderados, sob cada um dos enviesamentos utilizados, possibilitando uma análise multicriterial, comparativa e conjunta para esse estudo sistêmico que conduziu a hierarquização das alternativas, assim pode-se realizar a interpretação de sua maior ou menor conveniência para a solução a ser adotada. Os resultados das avaliações técnica, econômica e ambiental são apresentadas nas tabelas a seguir.

O estudo foi iniciado pelos critérios técnicos, considerando aspectos de infraestrutura e operação/manutenção, os resultados dessa etapa são expostos pela Tabela 3 a seguir. 
Tabela 3. Classificação das alternativas segundo critérios técnicos.

\begin{tabular}{|c|c|c|c|c|c|c|c|}
\hline \multirow{2}{*}{\multicolumn{2}{|c|}{ ASPECTO }} & \multirow{3}{*}{$\begin{array}{c}\text { PESO } \\
1\end{array}$} & \multicolumn{5}{|c|}{ ALTERNATIVA } \\
\hline & & & \multirow{2}{*}{$\begin{array}{l}1 \\
0\end{array}$} & \multirow{2}{*}{$\begin{array}{c}2 \\
10\end{array}$} & \multirow{2}{*}{\begin{tabular}{|c|}
3 \\
10
\end{tabular}} & \multirow{2}{*}{$\begin{array}{c}4 \\
10\end{array}$} & \multirow{2}{*}{$\begin{array}{c}5 \\
10\end{array}$} \\
\hline \multirow[b]{2}{*}{ Infraestrutura } & Necessidade de área & & & & & & \\
\hline & Risco de transbordamentos & 4 & 10 & 0 & 0 & 5 & 5 \\
\hline \multirow{3}{*}{ Operação/ manutenção } & Grau dificuldade & 2 & 10 & 0 & 10 & 10 & 10 \\
\hline & Risco lançamento de esgotos & 1 & 10 & 10 & 0 & 5 & 10 \\
\hline & Risco falha comportas & 2 & 10 & 5 & 5 & 5 & 5 \\
\hline \multicolumn{2}{|l|}{ Soma dos pesos } & 10 & 90 & 30 & 40 & 65 & 70 \\
\hline \multicolumn{2}{|l|}{ Nota } & & 9 & 3 & 4 & 6,5 & 7 \\
\hline \multicolumn{3}{|c|}{ CLASSIFICAÇÃO TÉCNICA } & $5^{a}$ & $1^{\mathrm{a}}$ & $2^{a}$ & $3^{a}$ & $4^{\mathrm{a}}$ \\
\hline
\end{tabular}

Fonte: Autores.

Conforme a Tabela 3 acima pode-se verificar que a Alternativa 1 (interligação com concordância de canais sem amortecimento) possui a classificação mais distante do ideal, mesmo que essa não necessite da aquisição de áreas para sua execução. Entretanto, a alternativa apresenta risco de transbordamentos, fator crucial para sua colocação em último lugar devido a relevância do aspecto possuir peso 4. Em primeiro lugar a Alternativa 2 (interligação com concordância de canais com amortecimento) destaca-se por possuir a menor soma dos pesos, isso ocorre em função do baixo risco de transbordamentos e da baixa dificuldade de operação/manutenção.

Após a avaliação técnica, foi realizada a avaliação econômica que pondera os custos de infraestrutura e operação/manutenção, seus resultados estão presentes na Tabela 4.

Tabela 4. Classificação das alternativas segundo critérios econômicos.

\begin{tabular}{|c|c|c|c|c|c|c|c|}
\hline \multirow{2}{*}{\multicolumn{2}{|c|}{ ASPECTO }} & \multirow{2}{*}{ PESO } & \multicolumn{5}{|c|}{ ALTERNATIVA } \\
\hline & & & 1 & 2 & 3 & 4 & 5 \\
\hline \multirow{3}{*}{ Custo de Instalação } & Aquisição de áreas & 1 & 0 & 10 & 10 & 5 & 5 \\
\hline & Implantação de canais & 2 & 0 & 0 & 0 & 10 & 10 \\
\hline & Volume de Acumulação & 2 & 0 & 5 & 10 & 10 & 10 \\
\hline \multirow{2}{*}{ Custo de Operação/Manutenção } & Operação & 2,5 & 10 & 0 & 10 & 10 & 10 \\
\hline & Manutenção & 2,5 & 10 & 0 & 10 & 10 & 10 \\
\hline Soma dos Pesos & & 10 & 50 & 20 & 80 & 95 & 95 \\
\hline Nota & & & 5 & 2 & 8 & 9,5 & 9,5 \\
\hline CLASSIFICAÇÃO ECONÔMICA & & & $2^{\mathrm{a}}$ & $1^{\mathrm{a}}$ & $3^{\mathrm{a}}$ & $4^{\mathrm{a}}$ & $4^{a}$ \\
\hline
\end{tabular}

Fonte: Autores.

Economicamente as Alternativas 4 e 5 não se encontram entre as melhores opções para a soluções da alagamentos por possuírem altos valores na soma dos seus pesos, de acordo com Tucci (2012) medidas estruturais apresentam os maiores custos e geralmente não são viáveis economicamente, a implantação de canais necessária nessas alternativas eleva significativamente o custo do projeto. Já a Alternativa 2, o primeiro lugar, seu único ponto negativo seria a aquisição de áreas, uma vez que o estudo está se referindo a uma área consolidada e extremamente urbanizada, contudo, a baixa soma dos seus pesos foram suficientes para mantê-la como a solução mais adequada.

O critério ambiental possui o maior peso dentre as avaliações e pondera aspectos de qualidade e quantidade da água, a Tabela 5 apresenta os resultados e classificação da análise. 
Research, Society and Development, v. 10, n. 11, e76101119352, 2021

(CC BY 4.0) | ISSN 2525-3409 | DOI: http://dx.doi.org/10.33448/rsd-v10i11.19352

Tabela 5. Classificação das alternativas segundo critérios ambientais.

\begin{tabular}{|c|c|c|c|c|c|c|c|}
\hline \multirow{2}{*}{\multicolumn{2}{|c|}{ ASPECTO }} & \multirow{3}{*}{$\begin{array}{c}\text { PESO } \\
5\end{array}$} & \multicolumn{5}{|c|}{ ALTERNATIVA } \\
\hline & & & \multirow{2}{*}{$\frac{\mathbf{1}}{10}$} & \multirow{2}{*}{$\frac{2}{10}$} & \multirow{2}{*}{$\frac{3}{10}$} & \multirow{2}{*}{$\frac{4}{10}$} & \multirow{2}{*}{$\frac{5}{10}$} \\
\hline Qualidade das águas & Pluviais com esgoto & & & & & & \\
\hline Quantidade das águas & Com alagamentos & 5 & 10 & 0 & 0 & 0 & 0 \\
\hline \multirow{3}{*}{\multicolumn{2}{|c|}{$\begin{array}{l}\text { Soma dos pesos } \\
\text { Nota } \\
\text { CLASSIFICAÇÃO AMBIENTAL }\end{array}$}} & 10 & 100 & 50 & 50 & 50 & 50 \\
\hline & & & 10 & 5 & 5 & 5 & 5 \\
\hline & & & $2^{\mathrm{a}}$ & $1^{\mathrm{a}}$ & $1^{\mathrm{a}}$ & $1^{\mathrm{a}}$ & $1^{\mathrm{a}}$ \\
\hline
\end{tabular}

Fonte: Autores.

A intensa urbanização pode gerar impactos sobre as águas urbanas, de acordo com Tucci (2012) pode ocorrer a perda de mananciais, a deterioração das águas e a diminuição da qualidade de vida da população, isso em decorrência de uma série de fatores como a disposição inadequada dos esgotos sanitários. Portanto, o aspecto de qualidade das águas pluviais com esgoto está altamente presente em todas as alternativas na Tabela 5, esse é um fator preocupante quando visamos o saneamento básico local. $\mathrm{O}$ aspecto quantidade de água pondera sobre a ocorrência de alagamentos, apenas a Alternativa 1 apresentou a ocorrência do evento, em vista disso, ela não se adequa ao objetivo do estudo.

Por fim, a classificação final das alternativas que foram avaliadas nas esferas técnica, econômica e ambiental está disposta na Tabela 6.

Tabela 6. Hierarquização e classificação geral das alternativas conforme critérios técnicos, econômicos e ambientais.

\begin{tabular}{|c|c|c|c|c|c|c|}
\hline \multirow{2}{*}{ AVALIAÇÃO } & \multirow{2}{*}{ PESO } & \multicolumn{5}{|c|}{ ALTERNATIVA } \\
\hline & & 1 & 2 & 3 & 4 & 5 \\
\hline Técnica & 3 & 9 & 3 & 4 & 6,5 & 7 \\
\hline Econômica & 3 & 5 & 2 & 8 & 9,5 & 9,5 \\
\hline Ambiental & 4 & 10 & 5 & 5 & 5 & 5 \\
\hline Soma dos pesos & 10 & 82 & 35 & 56 & 68 & 70 \\
\hline Nota & & 8,2 & 3,5 & 5,6 & 6,8 & 7 \\
\hline CLASSIFICAÇÃO GERAL & & $5^{\mathrm{a}}$ & $1^{\mathrm{a}}$ & $2^{\mathrm{a}}$ & $3^{\mathrm{a}}$ & $4^{a}$ \\
\hline
\end{tabular}

Fonte: Autores.

Na hierarquização apresentada na tabela acima a Alternativa 2 está como a melhor a solução para alagamentos na Sub-bacia 2 da BHEN, seus aspectos positivos estão entre baixo risco de alagamentos, baixo custo de operação/manutenção e a não necessidade de implantação de canais. Pode-se sugerir que o maior problema para a alternativa seja a necessidade de aquisição de áreas, há também a ocorrência de problemas como lançamento de esgoto e possíveis falhas de comportas, entretanto são fatores que podem ser contornados com medidas preventivas.

Figura 8 a seguir mostra graficamente a hierarquização apresentada na tabela acima, facilitando a visualização da hierarquização das alternativas estudadas. 
Research, Society and Development, v. 10, n. 11, e76101119352, 2021

(CC BY 4.0) | ISSN 2525-3409 | DOI: http://dx.doi.org/10.33448/rsd-v10i11.19352

Figura 8. Hierarquização das alternativas - quanto menor a nota, melhor a alternativa.

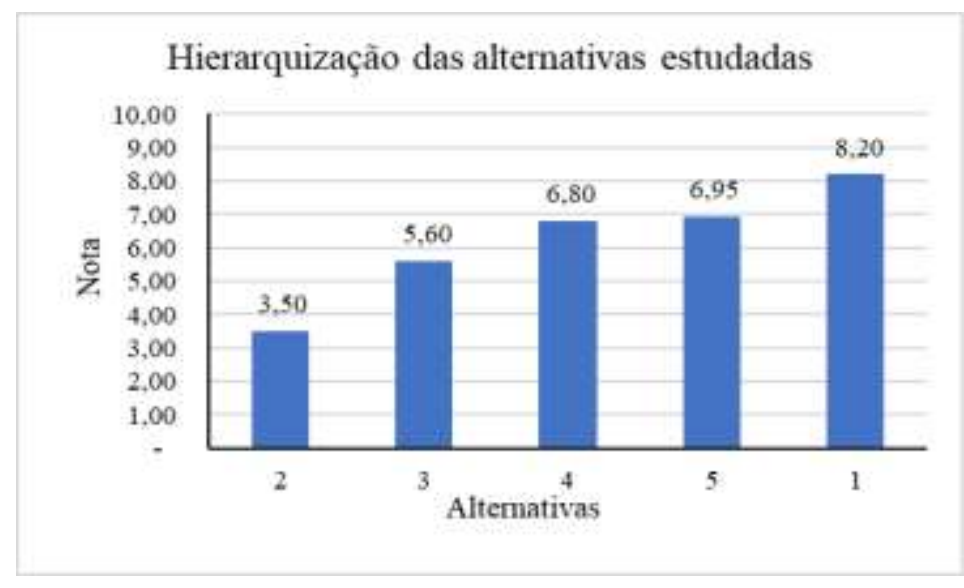

Fonte: Autores.

Observa-se na Tabela acima que a análise multicriterial aplicada resultou na seguinte hierarquização das alternativas, da melhor para a pior: $2^{\mathrm{a}}, 3^{\mathrm{a}}, 4^{\mathrm{a}}, 5^{\mathrm{a}}$ e $1^{\mathrm{a}}$.

\section{Considerações Finais}

Para buscar a melhor funcionalidade do sistema de macrodrenagem da Sub-bacia 2 da BHEN sob efeito de maré alta e precipitação com período de retorno de cinquenta anos, foram elaboradas com o auxílio do programa SWMM cinco alternativas. Buscou-se a melhor alternativa possível, utilizando-se de conceitos de boas práticas de Engenharia Consultiva, de modo a permitir o melhor resultado possível de funcionalidade da macrodrenagem da Sub bacia 2, permitindo interligar os canais implantados, de montante e de jusante, além de eliminar pontos de estrangulamento e de desassorear os canais revestidos, com importantes benefícios para toda a população residente nessa Sub bacia 2 pela minimização de alagamentos, bem como da trafegabilidade do sistema viário que sofre interrupção devido a esses alagamentos.

A análise multicriterial possibilitou a hierarquização das alternativas estudadas, através de uma análise geral, identificando-se a alternativa mais adequada levando-se em conta o conjunto dos aspectos técnicos, econômicos e ambientais, hierarquizando e classificando as alternativas, o que resultou na seguinte hierarquização das alternativas, da melhor para a pior: $2^{\mathrm{a}}, 3^{\mathrm{a}}, 4^{\mathrm{a}}, 5^{\mathrm{a}}$ e $1^{\mathrm{a}}$.

Conclui-se, portanto, que a análise multicriterial efetuada contribui firmemente para a seleção da melhor alternativa dentre as estudadas e implantar um sistema de macrodrenagem funcional, útil, adequado, seguro e inteligente, com grandes benefícios para a população beneficiada.

Para trabalhos futuros recomenda-se a aplicação da metodologia utilizada em outros sistemas de drenagem urbana da região amazônica, assim como, a realização de estudos integrando o software de modelagem hidrodinâmica, SWMM, com um Sistema de Informações Geográficas (SIG) para produção de projetos georreferenciados do local a ser estudado. Sugere-se também utilizar o método de lógica Fuzzy para aprimorar a análise tornando-a mais adequada às variáveis semânticas e numéricas.

\section{Agradecimentos}

O presente trabalho foi realizado com apoio da Coordenação de Aperfeiçoamento de Pessoal de Nível Superior -Brasil (CAPES) - Código de Financiamento 001. Agradece também ao Banco Interamericano de Desenvolvimento (BID) e ao 
Research, Society and Development, v. 10, n. 11, e76101119352, 2021

(CC BY 4.0) | ISSN 2525-3409 | DOI: http://dx.doi.org/10.33448/rsd-v10i11.19352

Programa de Saneamento da Bacia da Estrada Nova (PROMABEN) pelo apoio na presente pesquisa.

\section{Referências}

APCE. (2016). Prefeitura Municipal de Belém. Projeto do Sistema de Macrodrenagem: Reprogramação das Metas do Contrato de Repasse n 228.498-26/2008 FGTS/CAIXA/PAC Sub-bacia 02 da Bacia Hidrográfica da Estrada Nova - PROMABEN Assessoria, Projetos e Consultoria Ltda (APCE).

Abreu, G. S., \& Andrade, C. S. P. (2019). Geotecnologias Aplicadas à Caracterização da Temperatura da Superfície na Cidade de Teresina (PI). Acta Geográfica, 13 (32), 28-47.

Belém. (2008). Lei n. ${ }^{\circ} 8.655$, de 30 de julho de 2008. Dispõe sobre o Plano Diretor do Município de Belém, e dá outras providências. Diário Oficial do Município de Belém n. ${ }^{\circ} 11189.2^{\circ}$ caderno, p. 60-193.

Brito, D. S. (2006). Metodologia para seleção de alternativas de sistemas de drenagem. Dissertação de Mestrado em Tecnologia Ambiental e Recursos Hídricos, Universidade de Brasília, Brasília, Brasil.

Correa, D. L. (2020). Análise da susceptibilidade a inundações na bacia hidrográfica do Rio Uraim, Paragominas - PA. Dissertação de Mestrado em Recursos Hídricos, Universidade Federal do Pará, Belém. http://repositorio.ufpa.br/jspui/handle/2011/10751\&gt.

Castro, L. M. A. (2002). Proposição de indicadores para avaliação de sistemas de drenagem urbana. Dissertação de Mestrado em Saneamento, Meio Ambiente e Recursos Hídricos, Universidade Federal de Minas Gerais, Belo Horizonte, Brasil.

Castro, L. M. A. (2007). Proposição de metodologia para avaliação dos efeitos da urbanização nos corpos de água. Tese de Doutorado em Saneamento, Meio Ambiente e Recursos Hídricos Universidade Federal de Minas Gerais, Belo Horizonte, Brasil.

Canholi, A. P. (1999). O Plano Diretor de Macrodrenagem da Bacia do Alto Tietê. Estruturação geral e resultados iniciais. São Paulo: CBAT/Consórcio Enger-Promon-CKC/EPUSP.

ENGESOLO engenharia LTDA. (2008). Prefeitura Municipal de Belém. Elaboração de estudos e projetos básicos necessários à preparação do programa de macrodrenagem da Bacia da Estrada Nova - PROMABEN, Belo Horizonte.

Ferreira, A. M. (2019). Aplicação de ferramenta multicritério em um software de drenagem urbana para uso na previsão de eventos hidrológicos. Tese de Doutorado, Universidade de São Paulo, São Paulo, Brasil.

Instituto Brasileiro de Geografia e Estatística. (2016). Bases cartográficas 2016. http://ww.mapas.ibge.gov.br/bases-ereferenciais/basescartograficas/cartas.html.

Instituto Brasileiro de Geografia e Estatística. (2011). Censo Demográfico 2010, Área territorial brasileira. IBGE.

Instituto Brasileiro de Geografia e Estatística. (2019). Diretoria de Pesquisas, Coordenação de População e Indicadores Sociais, Estimativas da população residente com data de referência 01 de julho de 2019. IBGE.

Jannuzzi, P. de M., Miranda, W. L., \& Silva, D. S. G. da. (2009). Análise multicritério e tomada de decisão em políticas públicas: aspectos metodológicos, aplicativo operacional e aplicações. Informática Pública, 11(1), pp. 69-87.

Mendonça, E. C. (2009). Metodologia para avaliação de desempenho de sistemas de drenagem urbana. Dissertação de mestrado em Tecnologia Ambiental e Recursos Hídricos, Universidade de Brasília, Brasília, Brasil.

Prefeitura Municipal de Belém (2015). Dados da Cidade. Belém: PMB. www.belem.pa.gov.br/planodiretor/paginas/brasao.php.

Silva, T. C. (2016). Mapeamento das manchas de inundação para a cidade de Matias Barbosa- MG. Trabalho de Conclusão de Curso de Engenharia Ambiental e Sanitária, Universidade Federal de Juiz de Fora, Juiz de Fora, Minas Gerais, Brasil.

Souza, A. (2020). Lixo e enchente tomaram conta da rua Fernando Guilhon. https://www.oliberal.com/belem/chuva-forte-na-tarde-desta-segunda-feira-deixaruas-alagadas-1.240984.

Teixeira, G. S., Rodrigues, R. S. S., Crispim, D. L., Fernandes, L. L., \& Bittencourt, G. M. (2020). Metodologia para Caracterização e Avaliação de Sistemas de Drenagem urbana: uma revisão. Research, Society and Development, 9(4).

Tucci, C. E. M. (2012). Gestão da drenagem urbana. CEPAL/IPEA.

Villanueva, A. O. N., Tassi, R., Piccilli, D. G. A., Bemfica, D. C., \& Tucci, C. E. M. (2011). Gestão da drenagem urbana, da formulação à implementação. Revista de Gestão de Água da América Latina-REGA, 8(1), 5-18. 